\title{
ANXIETY AND DEPRESSION IN AT RISK PREGNANCIES: IMPACT ON PRENATAL ATTACHMENT
}

\section{F. Testouri ${ }^{1}$, M. Hamza ${ }^{2}$, A. Ben Amor ${ }^{3}$, M. Barhoumi ${ }^{1}$, R. Fakhfekh ${ }^{1}$, A. Triki ${ }^{3}$, A. Belhadj $^{2}$. ${ }^{1}$ University of Tunis EI Manar, Faculty of Medicine of Tunis, Tunisia ${ }^{2}$ Child and Adolescent Psychiatry Department, Mongi Slim Hospital ,Tunisia. ${ }^{3}$ Gynecology-obstetrics Department, Mongi Slim Hospital, Tunisia}

\section{Background and aims}

High risk pregnancies can be associated with increased levels of depression and anxiety. Researches indicate that maternal prenatal emotional well-being influences the maternal-fetal attachment (MFA) which has important implication in postnatal bonding. The aim of this study was to examine the influence of maternal prenatal anxiety and depression on MFA in high risk pregnancies.

\section{Methods}

A cross-sectional study was conducted between October 2017 and July 2018 on 95 high-risk women in their third trimester of pregnancy, hospitalized in a gynecology-obstetrics service in a public hospital in Tunis. The Hospital Anxiety and Depression Scale (HADS) was used to assess states of depression and anxiety. The Prenatal Attachment Inventory (PAI) was used for evaluation of MFA.

\section{Results and discussion}

\section{Sociodemographics characteristics $(\mathrm{n}=95)$ :}

Mean age: 31.18 years (18 to 45 years)

Education: university $28.4 \%$; high school $49.5 \%$

Socio-economic status: Low to medium: $89.5 \%$ Family support: $87.4 \%$

Disrupted marital relationship: $23 \%$

Physical and verbal abuse: $12.4 \%$

HADS MEAN SCORE $=17.4(\mathrm{SD}=7.6)$

Depression subscale mean score $=7.96$

Anxiety subscale mean score $=\mathbf{9 . 4 4}$

According to the HADS scores, the sample was divided into four groups: definite cases of anxiety and depression, anxiety without depression, depression without anxiety and a group without anxiety nor depression (55.8\%) (figure1).

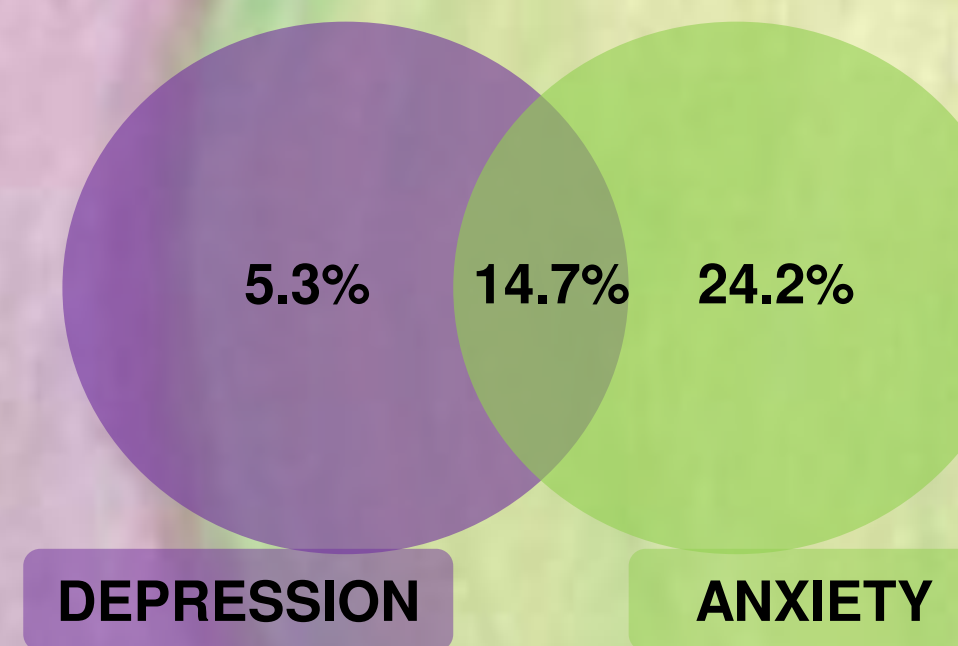

Figure 1: Pourcentage of cases of anxiety and depression among at risk pregnant women according to the HADS scores

Our study highlights high prevalence of anxiety-depressive disorders among pregnant women.

\section{Pregnancy Characteristics:}

Primiparity: $40 \%$

Planned pregnancy: $51.6 \%$

Wanted pregnancies: $87.4 \%$

Mean gestational time $=35.53$ weeks of amenorrhea

Fetal sex: F: $49.5 \% \mathrm{M}: 50.5 \%$

Hospital Stay: 4.83 days $(S D=7.17)$

\section{PAI MEAN SCORE $=\mathbf{5 7 . 2 1} \pm 9.04$}

The PAI scores correlated negatively and significantly with the HADS total score but when examining subscale scores, this correlation was found to be attributed to the depression dimension and not to the anxiety one (Table1).

PAl

Pearson's correlation $\quad$ P

\begin{tabular}{ccc}
\hline HADS score & -0.218 & $P$ \\
\hline Depression score & -0.205 & 0.034 \\
Anxiety score & -0.193 & 0.061 \\
\hline
\end{tabular}

Table 1: Correlation between anxiety-depressive disorders and prenatal attachment

Prenatal bonding is the result of a psychological dynamic that develops when the mother assimilates her maternal role and can be altered in case of mental disorders such as depression.

\section{Conclusion}

This study highlights the importance of assessing emotional wellbeing in at risk pregnancies especially depressive and anxiety symptoms given their influence on prenatal attachment. 